\title{
Tracking Attracting Lagrangian Coherent Structures in Flows
}

\author{
Dhanushka Kularatne \\ Drexel University \\ Philadelphia, Pennsylvania 19104
}

\author{
Ani Hsieh \\ Drexel University \\ Philadelphia, Pennsylvania 19104
}

\begin{abstract}
This paper presents a collaborative control strategy designed to enable a team of robots to track attracting Lagrangian coherent structures (LCS) and unstable manifolds in two-dimensional flows. Tracking LCS in dynamical systems is important for many applications such as planning energy optimal paths in the ocean and predicting various physical and biological processes in the ocean. Similar to existing approaches, the proposed strategy does not require global information about the dynamics of the surrounding flow, and is based on local sensing, prediction, and correction. Different from existing approaches, the proposed strategy has the ability to track attracting LCS and unstable manifolds in real time through direct computation of the local finite time Lyapunov exponent field. The collaborative control strategy is implemented on a team of robots and the theoretical guarantees of the tracking strategy is briefly discussed. We demonstrate the tracking strategy in simulation using static and time dependent flows and experimentally validate the strategy using a team of micro autonomous surface vehicles (mASVs) in an actual fluid environment.
\end{abstract}

\section{INTRODUCTION}

We are interested in the development of collaborative control strategies for distributed sensing and tracking of coherent structures and manifolds in flows using teams of autonomous underwater and surface vehicles (AUVs and ASVs). Researchers have shown that motion planning and adaptive sampling strategies for underwater robots can be improved when incorporating either historical ocean flow data [23, 25, 24] or multi-layer partial differential equation (PDE) models of the ocean [26, 12]. However, accessibility to and the overall quality of ocean current hindcasts, nowcasts, and forecasts provided by Navy Coastal Ocean Model (NCOM) databases [18], regional ocean model systems (ROMS) [25], and/or other numerical models are generally low. This is because most existing ocean models are derived from assimilated satellite and field data with predictions from numerical PDE models [21, 22]. As such, existing data sets that describe ocean flows are mostly finite time and of low spatio-temporal resolution.

In this work, we are interested in deploying teams of robots to track a class of coherent structures that are important for quantifying transport phenomena in flows. Inanc et al. showed that time and fuel optimal paths in the ocean can coincide with a specific class of coherent structures called Lagrangian coherent structures (LCS) [10, 19]. LCS are the extensions of stable and unstable manifolds to general timedependent flows [3, 2] and are similar to separatrices that divide the flow into dynamically distinct regions. This is supported by Forgoston et al.'s work where they showed that LCS coincide with regions in the flow field where more escape events occur [1]. As such, knowledge of LCS is important for planning energy efficient trajectories in the ocean, maintaining sensors in their desired monitoring regions [13, 9, 6], and enabling computationally tractable and efficient estimation and prediction of the underlying geophysical fluid dynamics.

In two-dimensional (2D) flows, LCS are one-dimensional separating boundaries analogous to ridges defined by local maximum instability, and can be quantified by local measures of Finite-Time Lyapunov Exponents (FTLE) [20]. The tracking of coherent structures in fluids is challenging since the structures are generally unstable and time-dependent. Existing work have shown that it is possible to use a team of robots to collaboratively track the stable manifolds and LCS boundaries in 2D flows [14, 16, 15]. The strategy relies on robots maintaining a boundary saddling formation while collecting local measurements of the flow velocity. LCS tracking is achieved by fusing the data to identify the region in the flow field with the extremal velocity. While the strategy has been validated using analytical models that describe large scale ocean circulation [8, 16], experimental data created in a flow tank, actual ocean data [14], and a scaled robotic platform [15], the work is primarily focused on the tracking of the repelling LCS boundaries or stable manifolds in 2D flows. Since the identification of repelling LCS boundaries requires the computation of forward FTLEs, real time tracking of the LCS boundaries in these existing work is achieved by solely examining the flow velocities in the region physically spanned by the robot team and do not rely on actual FTLE computations. As such, no information is obtained about the type of the LCS being tracked since tracking of the boundaries is not based on the local FTLE measures and the team may switch between tracking attracting and repelling LCS boundaries arbitrarily. Furthermore, since the LCS boundaries are not explicitly resolved the team of robots may end up tracking a non-existent boundary.

In this work, we present a methodology to track attracting LCS boundaries or unstable manifolds through the explicit on-board calculation of local FLTE fields. This can be done in real-time since the required FTLE calculations only use previously acquired flow velocity data. The proposed tracking strategy utilizes the FTLE field along with instantaneous local flow field measurements to resolve the attracting LCS bound- 
ary. In addition, we develop agent-level control policies which use the dynamics of the underlying flow field to maintain formation among a team of ASVs as they track the boundary. We verify the feasibility of the proposed method through simulations using 2D stationary and time varying flow fields and validate the proposed methodology in a laboratory setting using small ASVs in actual fluid flows.

The structure of the paper is as follows: We provide a background on the characterization of LCS using local FTLE measures and formulate the problem in Section III. The development of the proposed control strategy is presented in Section III and its theoretical feasibility is analyzed in Section IV. Simulation and experimental results are presented in Section $\mathrm{V}$ and Section VI respectively. We conclude with a discussion of our results and directions for future work in Section VII

\section{BACKGRound AND PRoblem STATEMENT}

\section{A. Background}

In this paper we consider the problem of tracking an attractive Lagrangian Coherent Structure (LCS) in a 2D planar flow field of the form

$$
\dot{\mathbf{x}}(t)=\mathbf{F}(\mathbf{x}, t)
$$

where $\mathbf{x}=[x, y]^{\prime}$ gives the position in the plane and $\mathbf{x}^{\prime}$ denotes the transpose of the vector $\mathbf{x}$. The boundary tracking methodology presented in this paper is based on the real time calculation of the FTLE field of (1) and the Proper Interior Maximum (PIM) triple inspired boundary tracking strategy [8].

The position of a fluid particle advected by the flow field given by (1), is a function of time $t$, the starting point of the particle $\mathbf{x}_{\mathbf{0}}$ and starting time $t_{0}$, i.e., $\mathbf{x}=\mathbf{x}\left(t ; \mathbf{x}_{\mathbf{0}}, t_{0}\right)$. Using the notation used by Shadden et al. [20], the solution to the dynamical system given in (1) can be viewed as a flow map which takes points from their initial position $\mathbf{x}_{\mathbf{0}}$ at time $t_{0}$ to their position at time $t$. This map, denoted by $\phi_{t_{0}}^{t}$, satisfies

$$
\phi_{t_{0}}^{t}\left(\mathbf{x}_{\mathbf{0}}\right)=\mathbf{x}\left(t ; \mathbf{x}_{\mathbf{0}}, t_{0}\right)
$$

and has the properties $\phi_{t_{0}}^{t_{0}}(\mathbf{x})=\mathbf{x}$ and $\phi_{t_{0}}^{s+t}(\mathbf{x})=\phi_{s}^{s+t}\left(\phi_{t_{0}}^{s}(\mathbf{x})\right)$. The Finite Time Lyaponov Exponent (FTLE) with a finite integration time interval $T$, associated with a point $\mathbf{x}$ at time $t_{0}$ is given by,

$$
\sigma_{t_{0}}^{T}(\mathbf{x})=\frac{1}{|T|} \ln \sqrt{\lambda_{\max }(\Delta)}
$$

where $\lambda_{\max }(\Delta)$ is the maximum eigenvalue of the finite-time version of the Cauchy-Green deformation tensor $\Delta$, given by,

$$
\Delta=\frac{d \phi_{t_{0}}^{t_{0}+T}(\mathbf{x})^{\prime}}{d \mathbf{x}} \frac{d \phi_{t_{0}}^{t_{0}+T}(\mathbf{x})}{d \mathbf{x}} .
$$

The value of $\Delta$ is computed numerically by discretizing the domain into a regular grid and computing the trajectories of each point and its immediate neighbors in the grid from time $t_{0}$ to $t_{0}+T$ (see Fig 1(a) . For each point in the grid, the trajectories are computed by numerically integrating (1) from $t_{0}$ to $t_{0}+T$.
In this paper, LCS are considered to be ridges in the FTLE field calculated using (3), i.e., maximal values in the FTLE field as defined by Shadden et al. [20]. The forward-time FTLE field calculated by advecting fluid particles forward in time $(T>0)$, reveals repelling LCS which are analogous to the stable manifolds of saddle points in a time independent flow field. Conversely, the backward-time FTLE field $(T<0)$ reveals attracting LCS which are analogous to unstable manifolds of a time independent flow field.

The FTLE value gives a measure of the maximum expansion of two initially nearby particles when they are advected by the flow. Therefore, particles initiated on opposite sides of an LCS will have much higher FTLE values than their neighbors, since an LCS acts as a boundary between two dynamically distinct regions of the flow [20]. Thus, by calculating the FTLE field in a neighborhood that contains an LCS, it should be possible to find the LCS by tracing out points with the highest FTLE values.

\section{B. Problem Statement}

Let $B_{u}$ denote an attractive LCS of the flow map given in (11). In this work, a team of $N$ Autonomous Surface Vehicles (ASVs) are used to track $B_{u}$ in real time. Each ASV is assumed to have the kinematic model given by,

$$
\begin{aligned}
& \dot{x}_{i}=V_{i} \cos \theta_{i}+u_{i} \\
& \dot{y}_{i}=V_{i} \sin \theta_{i}+v_{i}
\end{aligned}
$$

where $V_{i}$ is the speed of the ASV with respect to the flow, $\theta_{i}$ is the heading direction of the ASV, $\mathbf{x}_{i}=\left[x_{i}, y_{i}\right]^{\prime}$ is the position of the ASV in the global frame and $\mathbf{u}_{i}=\left[u_{i}, v_{i}\right]^{\prime}$ is the velocity of the underlying flow field at $\mathbf{x}_{i}$. Specifically, $u_{i}=F_{x}\left(\mathbf{x}_{i}, t\right)$ and $v_{i}=F_{y}\left(\mathbf{x}_{i}, t\right)$. In vector form, the velocity of an ASV could be written as,

$$
\mathbf{V}_{\mathbf{g i}}=\mathbf{V}_{\mathbf{i} / \mathbf{f}}+\mathbf{F}\left(\mathbf{x}_{\mathbf{i}}, t\right)
$$

where $\mathbf{V}_{\mathbf{g i}}=[\dot{x}, \dot{y}]^{\prime}$ and $\mathbf{V}_{\mathbf{i} / \mathbf{f}}$ is the velocity of the $i^{\text {th }}$ ASV relative to the flow. Note that $V_{i}$ and $\theta_{i}$ are the magnitude and direction of $\mathbf{V}_{\mathbf{i} / \mathbf{f}}$ respectively. The control inputs of the $i^{\text {th }}$ ASV are $V_{i}$ and $\omega_{i}=\dot{\theta}_{i}$. We assume that each ASV has the capability to measure the flow velocity at its current position and that each ASV can communicate bi-directionally with a data processing vehicle. Throughout this work, we assume that the ASVs are initialized as a grid with the center ASV $\left(P_{c}\right)$, coincident on $B_{u}$ as shown in Fig. 1(b). Using the definitions and assumptions given above, we can now state the problem being addressed in this paper as follows:

"Given an initial estimate for the position of a point on $B_{u}$ at time $t_{0}$, develop a collaborative control strategy to track $B_{u}$ for time $t>t_{0}$ for a team of ASVs with kinematic models given by (5) while maintaining a grid formation similar to Fig. 1(b)].

\section{Methodology}

\section{A. Manifold Tracking}

The methodology proposed in this paper utilizes a combination of backward-time FTLE computations and the PIM 


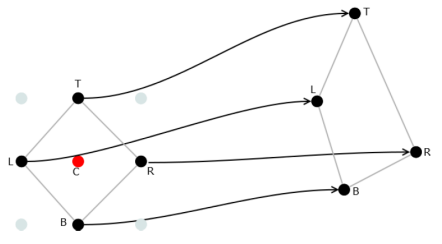

(a)

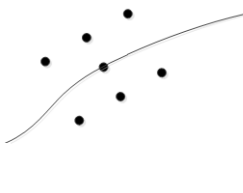

(b)
Fig. 1. (a) Locations of neighboring particles for the numerical computation of $\Delta$. (b) Agents are initialized in a grid formation with the center of the grid coincident on the attracting LCS

triple inspired tracking strategy [8] to locate the attractive LCS boundary. Since LCS are time varying, tracking based solely on FTLE computations will tend to fail especially when the time scales of the past flow velocity measurements are not representative of the time scales of the current or future time variations of the boundary.

1) Locating the LCS boundary using the FTLE field: Considering the flow velocity measurements obtained by the team of ASVs, an approximation could be obtained for the velocity field given in (1) as

$$
\dot{\hat{\mathbf{x}}}(t)=\hat{\mathbf{F}}(\mathbf{x}, t)
$$

for $t=t_{0}, \ldots, t_{i}, \ldots, t_{f}$ and $\mathbf{x}=\mathbf{x}_{\mathbf{1}}^{\mathbf{0}}, \mathbf{x}_{\mathbf{2}}^{\mathbf{0}}, \ldots, \mathbf{x}_{\mathbf{N}}^{\mathbf{0}}, \ldots, \mathbf{x}_{\mathbf{1}}^{\mathbf{f}}, \mathbf{x}_{\mathbf{2}}^{\mathbf{f}}, \ldots, \mathbf{x}_{\mathbf{N}}^{\mathbf{f}}$, where $t_{i}$ is the $i^{t h}$ sampling instance and $\mathbf{x}_{\mathbf{j}}^{\mathbf{i}}$ is the position of the $j^{t h} \mathrm{ASV}$ at $t_{i}$. The trajectories of a set of particles arranged in a grid around the center ASV $P_{c}$, are computed by integrating $\hat{\mathbf{F}}$ backwards in time, i.e., $T<0$. The resulting flow map is used in (4) and (3) to compute the backwardtime FTLE on this grid. However, the problem of using $\hat{\mathbf{F}}$ to compute the backward time FTLE is its inherent sparseness. Therefore, trilinear interpolation is used on $\hat{\mathbf{F}}$ to approximate the flow velocities at spatio-temporal coordinates for which direct velocity measurements are unavailable. It has been found that FTLE maxima are relatively insensitive to the interpolation scheme used for the computations [5]. Since we assume that $P_{c}$ is initialized on $B_{u}$ itself, following from the discussion in Section II-A the point with the highest FTLE value on this grid will correspond to a point on the LCS. Let this boundary location found using the FTLE field be denoted by $\mathbf{q F}_{\mathbf{F}}$.

2) Locating the LCS boundary using the PIM inspired strategy: This boundary tracking strategy introduced by Hsieh et al. [8] is based on the PIM triple method [17]. Given a team of three robots, denoted as $\{L ; C ; R\}$, robot $\mathrm{C}$ is tasked to remain close to the boundary of interest, $B_{u}$. Robots $\mathrm{L}$ and R are tasked to remain on opposite sides of $B_{u}$ at all times and thus maintain a saddle straddle formation. Let $\hat{\mathbf{u}}_{\mathbf{L}}(t), \hat{\mathbf{u}}_{\mathbf{C}}(t)$ and $\hat{\mathbf{u}}_{\mathbf{R}}(t)$ denote the flow velocity measurements obtained by $\mathrm{L}, \mathrm{C}$ and $\mathrm{R}$ at their respective positions $\mathbf{x}_{\mathbf{L}}(t), \mathbf{x}_{\mathbf{C}}(t)$ and $\mathbf{x}_{\mathbf{R}}(t)$. The saddle straddle line segment $J$ whose end points are defined by $\mathbf{x}_{\mathbf{L}}$ and $\mathbf{x}_{\mathbf{R}}$, is discretized such that $\mathbf{x}_{\mathbf{L}}=\mathbf{q}_{\mathbf{1}}<$ $\mathbf{q}_{2}<\ldots<\mathbf{q}_{\mathbf{M}}=\mathbf{x}_{\mathbf{R}}$. Given a set of velocity measurements $\hat{\mathbf{u}}_{\mathbf{i}}(t)$ and corresponding position estimates $\hat{\mathbf{x}}_{\mathbf{i}}(t)$, the flow velocity at $\mathbf{q}_{\mathbf{k}}$ is calculated as $\mathbf{u}\left(\mathbf{q}_{\mathbf{k}}\right)=\sum_{j} \sum_{i=1}^{N} \frac{w_{i j} \hat{\mathbf{u}}_{\mathbf{i}}(j)}{\sum_{j} \sum_{i=1}^{N} w_{i j}}$ where $w_{i j}=\left\|\hat{\mathbf{x}}_{\mathbf{i}}(j)-\mathbf{q}_{\mathbf{k}}\right\|^{-2}$. The location of the boundary on the current saddle straddle line segment is given by the point $\mathbf{q}_{\mathbf{B}}=\underset{k=1 \ldots, M}{\arg \max } \mathbf{u}\left(\mathbf{q}_{\mathbf{k}}\right)^{\prime} \hat{\mathbf{u}}_{\mathbf{c}}(t)$. For more information on this boundary tracking method, interested readers are referred to [8, 14].

This method is used to compute the boundary location on a saddle straddle line segment defined by the two immediate neighbors of $P_{c}$ on either side of the boundary. Let this boundary location found using the PIM triple based method be denoted by $\mathbf{q}_{\mathbf{P}}$.

3) Boundary tracking: The current location of the LCS $\left(\mathbf{q}_{\mathbf{h}}\right)$ is considered to be the weighted average of $\mathbf{q}_{\mathbf{F}}$ and $\mathbf{q}_{\mathbf{P}}$. As such

$$
\mathbf{q}_{\mathbf{h}}=\frac{d_{P} \mathbf{q}_{\mathbf{F}}+d_{F} \mathbf{q} \mathbf{P}}{d_{P}+d_{F}}
$$

where $d_{F}$ and $d_{P}$ are shortest distance from $B_{u}$ to $\mathbf{q}_{\mathbf{F}}$ and $\mathbf{q}_{\mathbf{P}}$ respectively.

Next, the Lagrangian nature of the FLTE field is used to predict the future location of the LCS boundary. It has been shown by Shadden et al. [20] that FTLE values calculated with large integration (advection) times are constant along trajectories. Therefore, to get an approximation of the future LCS location $\hat{\mathbf{q}}_{\mathbf{h}}$, we advect a fluid particle located at $\mathbf{q}_{\mathbf{h}}$ forward in time for $T_{1}$ seconds using $\hat{\mathbf{F}}$. Fig. 2(a) gives an illustration of the process.

In order for $P_{c}$ to follow $B_{u}$, we want $P_{c}$ to head towards $\hat{\mathbf{q}}_{\mathbf{h}}$ at all times, i.e., we want the velocity of $P_{c}$ in the global frame, $\mathbf{V}_{\mathbf{g c}}$, to be directed towards $\hat{\mathbf{q}}_{\mathbf{h}}$ at all times. As shown in Fig. 2(b) this could be done by setting $\mathbf{V}_{\mathbf{c} / \mathbf{f}}$ (velocity of $P_{c}$ relative to the flow) appropriately. Since we have the freedom to set $\left|\mathbf{V}_{\mathbf{c} / \mathbf{f}}\right|$ arbitrarily and set the direction $\theta_{d}$ accordingly, we select the minimum possible value for $\left|\mathbf{V}_{\mathbf{c} / \mathbf{f}}\right|$. It can be shown that the minimum possible $\left|\mathbf{V}_{\mathbf{c} / \mathbf{f}}\right|$ and the corresponding $\theta_{d}$ are given by,

$$
\begin{aligned}
\left|\mathbf{V}_{\mathbf{c} / \mathbf{f}}\right| & =\left|v_{i} \cos \alpha-u_{i} \sin \alpha\right| \\
\theta_{d} & =\alpha+\operatorname{sign}\left(\mathbf{F}\left(\mathbf{x}_{\mathbf{c}}, t\right) \times \mathbf{V}_{\mathbf{g c}}\right) \frac{\pi}{2}
\end{aligned}
$$

where $\alpha$ is the direction of $\mathbf{V}_{\mathbf{g c}}$ as shown in Fig. 2(b) Using (9), the velocity commands for $P_{c}$ are set as,

$$
\begin{aligned}
V_{c} & =\left|\hat{v}_{i} \cos \alpha-\hat{u}_{i} \sin \alpha\right| \\
\omega_{c} & =K_{\omega}\left(\theta_{d}-\theta_{c}\right)+\dot{\theta}_{d}
\end{aligned}
$$

where $\hat{u}_{i}=\hat{F}_{x}\left(\mathbf{x}_{\mathbf{c}}, t\right), \hat{v}_{i}=\hat{F}_{y}\left(\mathbf{x}_{\mathbf{c}}, t\right)$ and $\theta_{c}$ is the current heading angle of $P_{c}$. Setting the velocity commands as in 10 will result in $\mathbf{V}_{\mathbf{c} / \mathbf{f}}$ attaining the values given in 9 and as a consequence $P_{c}$ will always be headed towards $\hat{\mathbf{q}}_{\mathbf{h}}$.

Remark 1: The proposed tracking strategy fundamentally relies on the team's ability to simultaneously measure the flow field on opposite sides of $B_{u}$. Thus, it is possible track the LCS using a minimum of three agents. However, the spatial spread of the flow data collected with just three agents would not be sufficient for effective FTLE calculations. 


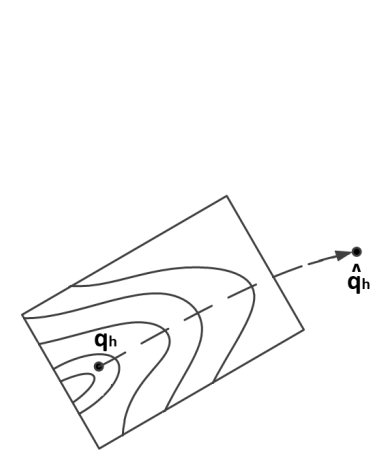

(a)

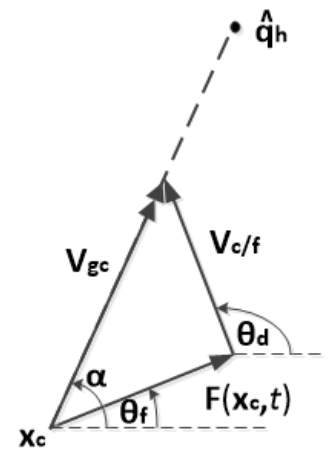

(b)
Fig. 2. (a) The LCS location $\mathbf{q}_{\mathbf{h}}$ is detected on the calculated FTLE grid and the flow map is used to predict the future LCS location $\hat{\mathbf{q}}_{\mathbf{h}}$. (b) $\mathbf{V}_{\mathbf{c} / \mathbf{f}}$ is selected such that $\mathbf{V}_{\mathbf{g c}}$ is always directed towards $\hat{\mathbf{q}}_{\mathbf{h}}$

\section{B. Formation Keeping}

It has been shown by Haller et al. [3] that particle trajectories which are infinitesimally close to the attracting coherent structure, will separate from the manifold at an exponential rate when time is propagated backwards. In other words, particle trajectories which are close enough to the attracting LCS $\left(B_{u}\right)$, will converge towards $B_{u}$ at an exponential rate in forward time. We make use of this property of the attracting LCS to design a control strategy that will maintain the initial grid formation of the ASVs. In this formulation we assume that $P_{c}$ tracks $B_{u}$ accurately using the methodology provided in Section III-A and that the path of $P_{c}$ provides the trajectory of $B_{u}$.

Since the path that the formation of ASVs needs to follow is defined by the boundary $B_{u}$, we consider the tangent and normal directions of $B_{u}$ in the development of the proposed control strategy. At any point on $B_{u}$, the tangent direction is defined as the direction in which a fluid particle would propagate, when it is placed at that point. The normal direction to $B_{u}$ is considered to be pointing towards its instantaneous center of rotation. Let $\hat{\mathbf{t}}$ be the unit vector in the tangent direction and $\hat{\mathbf{n}}$ be the unit vector in the normal direction. Utilizing this notation, the flow velocity at a point $\mathbf{x}$ near $B_{u}$ could be written as,

$$
\mathbf{F}(\mathbf{x}, t)=V_{t f}(d) \hat{\mathbf{t}}+V_{n f}(d) \hat{\mathbf{n}}
$$

where $d$ is the normal distance measured from $B_{u}$ to $\mathbf{x} . d$ is considered to be positive when measured in the direction of $\hat{\mathbf{n}}$. Similarly, the velocity of the $i^{\text {th }}$ ASV relative to the flow, $\mathbf{V}_{\mathbf{i} / \mathbf{f}}$, could also be expressed as,

$$
\mathbf{V}_{\mathbf{i} / \mathbf{f}}=V_{t i}(d) \hat{\mathbf{t}}+V_{n i}(d) \hat{\mathbf{n}}
$$

Using (11) and 12, 6 can be rewritten in terms of $\hat{\mathbf{t}}$ and $\hat{\mathbf{n}}$ as,

$$
\mathbf{V}_{\mathbf{g i}}=V_{g i}^{t} \hat{\mathbf{t}}+V_{g i}^{n} \hat{\mathbf{n}}
$$

where

$$
\begin{gathered}
V_{g i}^{t}=V_{t i}(d)+V_{t f}(d) \\
V_{g i}^{n}=V_{n i}(d)+V_{n f}(d)
\end{gathered}
$$

$V_{g i}^{t}$ governs the motion of the ASV parallel to $B_{u}$ while $V_{g i}^{n}$ governs the motion perpendicular to $B_{u}$. Since it is assumed that $B_{u}$ is provided by the path of $P_{c}, V_{g i}^{t}$ also governs the motion of the ASV parallel to $P_{c}$ and $V_{g i}^{n}$ governs the normal distance from $P_{c}$ to the ASV. For the ASVs to remain in grid formation around $P_{c}, V_{g i}^{t}$ should make the $i^{t h} \mathrm{ASV}$ follow $P_{c}$ in the $\hat{\mathbf{t}}$ direction while $V_{g i}^{n}$ should make the ASV maintain a fixed distance from $P_{c}$ in the $\hat{\mathbf{n}}$ direction.

In order to ensure that the ASVs follow $P_{c}$ in the $\hat{\mathbf{t}}$ direction, we want,

$$
V_{t i}(d)=\left|\mathbf{V}_{\mathbf{g c}}\right|\left(\frac{\rho-d}{\rho}\right)-\hat{\mathbf{F}}\left(\mathbf{x}_{\mathbf{i}}, t\right) \cdot \hat{\mathbf{t}}
$$

where $\left|\mathbf{V}_{\mathbf{g c}}\right|$ is the speed of $P_{c}$ in the global frame and $\rho$ is the radius curvature of $B_{u}$ at the current location of $P_{c}$. If $V_{t i}(d)$ attains the value in 15 , then $V_{g i}^{t}$ in $14 \mathrm{a}$ would be set to,

$$
V_{g i}^{t}=\left|\mathbf{V}_{\mathbf{g c}}\right|\left(\frac{\rho-d}{\rho}\right)
$$

which would ensure that the $i^{t h} \mathrm{ASV}$ would follow $P_{c}$ in the $\hat{\mathbf{t}}$ direction. Let's denote this desired value of $V_{t i}$ given in 15 as $\bar{V}_{t i}$.

The converging property of $B_{u}$ near its boundary is used to find a velocity profile for $V_{n i}(d)$ that guarantees that an ASV will maintain a fixed normal distance from $P_{c}$. The observation of exponential convergence of particle trajectories near $B_{u}$ made in [3], suggests that $\left|V_{n f}\right|$ increases linearly with $|d|$, at points very close to $B_{u}$. Therefore it is assumed that,

$$
V_{n f}(d)=-\lambda d \quad, \quad-d_{l^{-}} \leq d \leq d_{l^{+}}
$$

where $\left[-d_{l^{-}}, d_{l^{+}}\right]$defines the attracting region of $B_{u}$ and $\lambda(>$ $0)$ defines the rate of convergence of fluid particles towards $B_{u}$. Such a flow velocity profile would make all fluid particles in the interval $\left[-d_{l^{-}}, d_{l^{+}}\right]$converge to $B_{u}$ exponentially. Since the ASVs are required to maintain a fixed normal distance to $B_{u}, V_{n i}(d)$ needs to be set such that the ASVs converge to a boundary parallel to $B_{u}$, instead to $B_{u}$ itself. Therefore $V_{n i}(d)$ is set to

$$
V_{n i}(d)=\frac{\beta}{d} \quad-d_{l^{-}} \leq d \leq d_{l^{+}}
$$

where $\beta$ is a design parameter. Let's denote this desired value of $V_{n i}$ given in 18 as $\bar{V}_{n i}$. Fig. 3 shows the velocity profiles of $V_{n f}, \bar{V}_{n i}$ and their sum $V_{g i}^{n}$ in the attracting region of $B_{u}$. Note that there is a discontinuity at $d=0$ in the profiles of $\bar{V}_{n i}$ and $V_{g i}^{n}$. This does not pose a problem because the ASVs do not operate at $d=0$ as we show in the next section.

In order to attain the desired values for $V_{t i}(d)$ and $V_{n i}(d)$ given in (15) and (18) respectively, the control inputs of the $i^{\text {th }} \mathrm{ASV}$ are set as

$$
\begin{aligned}
V_{i} & =\sqrt{\bar{V}_{t i}^{2}+\bar{V}_{n i}^{2}} \\
\omega_{i} & =K_{\omega i}\left(\theta_{d i}-\theta_{i}\right)+\dot{\theta}_{d i}
\end{aligned}
$$




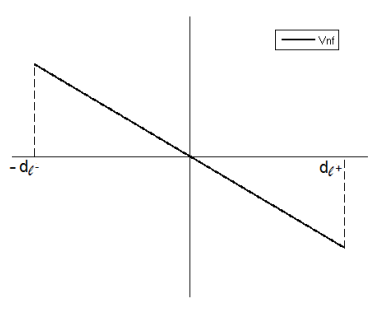

(a)

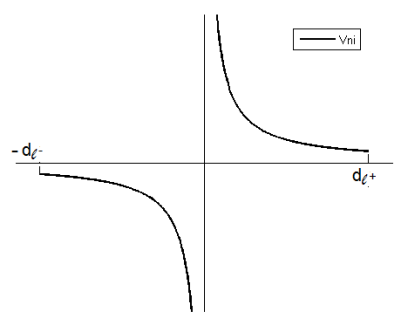

(b)

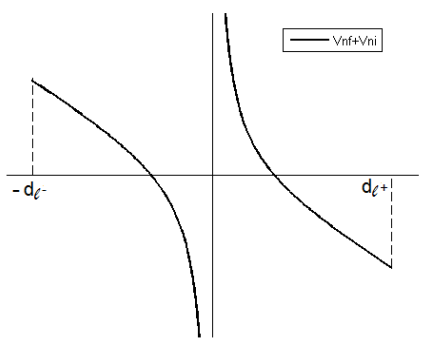

(c)

Fig. 3. Velocity profiles of (a) $V_{n f}(d)$, (b) $V_{n i}(d)$ and (c) $V_{g i}^{n}(d)=V_{n f}(d)+$ $V_{n i}(d)$.

with $\theta_{d i}=\operatorname{atan} 2\left(\bar{V}_{n i}, \bar{V}_{t i}\right)+\alpha$, where $\alpha$ is the heading angle of $P_{c}$.

Remark 2: The formation keeping strategy outlined above is based on local information. Therefore, the control commands for each ASV is computed individually. In the manifold tracking strategy, the tracking agent uses flow velocity measurements obtained by the other agents to compute the local FTLE field. While the computation of the local FTLE field is currently centralized, the process can be distributed since the computations required for the calculation of the FTLE field can be parallelized or carried out in a distributed fashion.

\section{ANALYSIS}

In this section, we discuss how setting $V_{t i}$ and $V_{n i}$ to their desired values given in 15 and 18 will result in the $i^{\text {th }}$ ASV maintaining its formation with respect to the center ASV $P_{c}$. As shown in (16), setting $V_{t i}$ as in 15 will result in the $i^{t h}$ ASV following $P_{c}$ in the $\hat{\mathbf{t}}$ direction. We are more interested in how setting $V_{n i}$ as in 18 results in the $i^{\text {th }}$ ASV maintaining a fixed distance from $B_{u}$.

As discussed in the previous section, the normal component of the velocity of the $i^{t h} \mathrm{ASV}$ in the global frame, $V_{g i}^{n}$ given in 14b), governs the normal distance from $B_{u}$ to the ASV. Specifically, we could say that $\dot{d}=V_{g i}^{n}(d)$. Substituting for $V_{n f}$ and $V_{n i}$ in $V_{g i}^{n}$ gives,

$$
\dot{d}=\frac{\beta}{d}-\lambda d \quad, \quad-d_{l^{-}} \leq d \leq d_{l^{+}}
$$

Solving 201 gives,

$$
d=\left\{\begin{array}{l}
\sqrt{\left(d_{0}^{2}-\frac{\beta}{\lambda}\right) e^{-2 \lambda t}+\frac{\beta}{\lambda}} \quad d_{0}>0 \\
-\sqrt{\left(d_{0}^{2}-\frac{\beta}{\lambda}\right) e^{-2 \lambda t}+\frac{\beta}{\lambda}} \quad d_{0}<0
\end{array}\right.
$$

where $d_{0}$ is the initial distance from $B_{u}$ to the ASV. From 21, it is easy to see that,

$$
\lim _{t \rightarrow \infty} d=\left\{\begin{array}{cc}
\sqrt{\frac{\beta}{\lambda}}, & d_{0}>0 \\
-\sqrt{\frac{\beta}{\lambda}}, & d_{0}<0
\end{array}\right.
$$

Thus it can be seen that $d$ will always stabilize to $\pm \sqrt{\frac{\beta}{\lambda}}$ and it will never approach $d=0$.

\section{Simulation Results}

In this section we validate the proposed tracking and formation keeping strategy in simulations. In all the simulations, we employ a team of seven robots to track the attracting LCS. The strategy is evaluated in 2D static conservative flows, 2D time-varying conservative flows and 2D periodic flows.

\section{A. 2D Static Conservative Flows}

In these simulations, the proposed methodology is used to track static boundaries in conservative flows. The flow velocity in this case is given by,

$$
F=a \nabla \varphi-b \nabla \times \psi
$$

where $a, b>0$ and $\varphi$ is an artificial potential function such that $\varphi(\mathbf{x})=0$ for all $\mathbf{x} \in B_{u}$ and $\varphi(\mathbf{x})<0$ for any $\mathbf{x} \in \mathbb{R}^{2} / B_{u}$. The vector $\psi$ is a $3 \times 1$ vector whose entries are given by $[0,0, \gamma(x, y)]^{T}$ where $\gamma(x, y)$ is the curve describing the desired boundary [7]. Selecting $a>0$ ensures that the flow field $\mathbf{F}$ has an attracting LCS.

Fig. 4(a) shows a team of seven ASVs tracking a circular attracting LCS and Fig 4(b) shows a team of seven ASVs tracking a sinusoidal attracting LCS. In both cases, the actual LCS is shown in red while the detected LCS, i.e., the trajectory of $P_{c}$, is shown in blue.

It can be seen that $P_{c}$ tracks the boundary accurately in both cases. The mean tracking errors for the circular and sinusoidal boundaries were found to be 2.67 and 4.59 respectively. The mean tracking error represents the average normal distance from $P_{c}$ to the actual boundary. Compared with the spatial spread of the boundaries, the tracking errors are relatively small. As shown in section III-A, the future LCS location is predicted by projecting the current LCS location forward in time using past velocity data. Therefore, tracking errors can be expected if the past flow velocity data does not provide an indication of the future curvature of the boundary. 
TABLE I

THE MEASURED AND EXPECTED DISTANCE FROM AN ASV TO THE BOUNDARY

\begin{tabular}{|c|c|c|c|c|}
\hline Boundary Type & $\lambda$ & $\beta$ & $\mathbf{d}_{\exp }$ & $\mathbf{d}_{\text {act }}$ \\
\hline Circular & 0.4 & 250 & 25 & 25.1 \\
\hline Sinusoidal & 0.1 & 40 & 20 & 19.7 \\
\hline
\end{tabular}

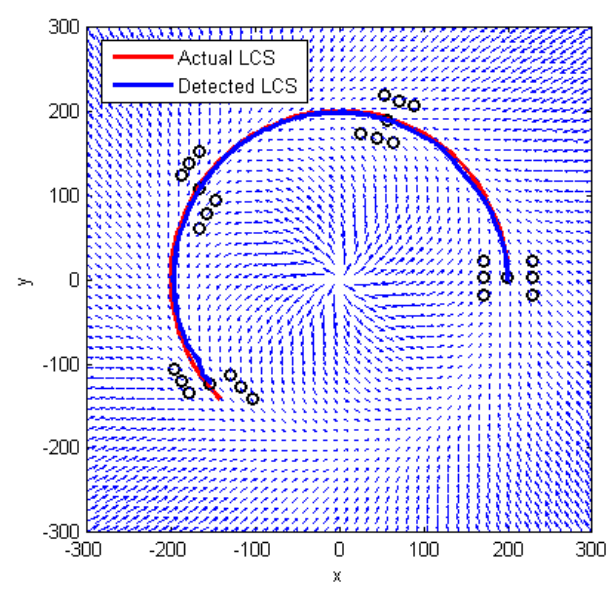

(a)

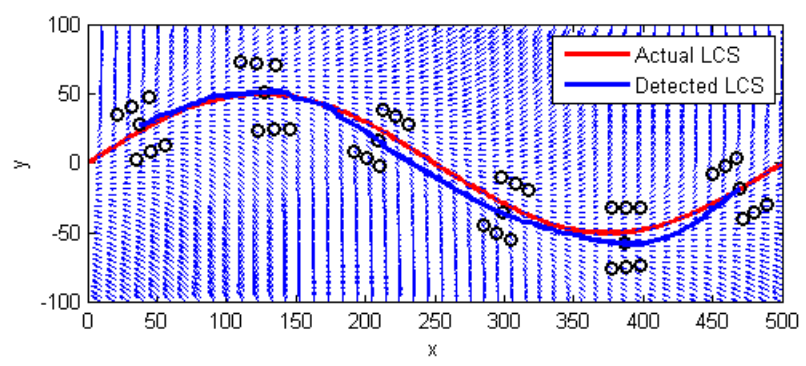

(b)

Fig. 4. (a) Shows the progression of the ASVs while tracking a circular boundary; (b) shows the progression of the ASVs while tracking a sinusoidal boundary.

In both cases, the team maintains formation while tracking the boundary. Table I shows the average distance an ASV maintains from the boundary in each case. In Table I] $\lambda, \beta$ represent the corresponding values in (17) and (18) respectively, $d_{\text {exp }}$ is the expected normal distance from the boundary to an ASV calculated using (22) and $d_{a c t}$ is the average distance an ASV maintains from the boundary in the simulations. Note that in both cases $\lambda$ was calculated using a linear approximation of the flow near the boundary.

\section{B. 2D Time-Varying Conservative Flows}

In this simulation we consider a conservative flow field in which the attracting LCS is time varying. The flow field used in this case has the same form as (23), with time varying $\varphi$ and $\gamma$. Fig. 5 shows the locations of a team of ASVs tasked with tracking a time varying sinusoidal front in a conservative flow field. The background shows the actual FTLE field computed using complete knowledge of the flow field. The dark red areas in the FTLE field indicate the attracting LCS boundary. Fig. 5 shows that $P_{c}$ tracks the time-varying LCS accurately and the formation of the ASVs is maintained through the tracking process.

\section{Time-Dependent Wind-Driven Double-Gyre Flow}

In this section we consider a flow field where $\mathbf{F}$ is determined by the wind-driven double-gyre flow model, given by

$$
\begin{aligned}
\dot{x} & =-\pi A \cos \left(\pi \frac{f(x, t)}{s}\right) \sin \left(\pi \frac{y}{s}\right) \frac{d f}{d x}-\mu x, \\
\dot{y} & =\pi A \sin \left(\pi \frac{f(x, t)}{s}\right) \cos \left(\pi \frac{y}{s}\right)-\mu y, \\
f(x, t) & =\varepsilon \sin (\omega t+\psi) x^{2}+(1-2 \varepsilon \sin (\omega t+\psi)) x .
\end{aligned}
$$

When $\varepsilon=0$, the double-gyre flow is time-independent, while for $\varepsilon \neq 0$, the gyres undergo a periodic expansion and contraction in the $y$ direction. In 24], $A$ approximately determines the amplitude of the velocity vectors, $\omega / 2 \pi$ gives the oscillation frequency, $\varepsilon$ determines the amplitude of the up-down motion of the separatrix between the gyres, $\psi$ is the phase, $\mu$ determines the dissipation and $s$ scales the dimensions of the workspace.

Fig. 6 shows positions of a team of ASVs tasked with tracking an attractive LCS in a time-varying double-gyre flow. It can be seen that the team of ASVs are able to track the LCS boundary accurately while maintaining the desired formation of the agents.

\section{LCS tracking in the presence of measurement noise}

In this section, the proposed tracking and formation keeping strategy is evaluated in the presence of measurement noise. Zero mean Gaussian noise, with standard deviation $\sigma=\sqrt{2 I}$ with noise intensity I, is added to the velocity measurements obtained by each ASV to simulate measurement noise. Fig. 7 shows a team of ASVs tracking the LCS in a time-varying conservative flow in the presence of measurement noise. In this case, the noise intensity is set to 0.25 which results in a standard deviation for the measurement noise which is approximately $12 \%$ of the mean flow velocity on the LCS boundary. Comparison with Fig. 5 shows that there is no significant degradation of the boundary tracking performance even in the presence of measurement noise. This is expected since it has been shown that LCS extracted from FTLE fields are robust to measurement errors of the velocity field [5, 4].

\section{EXPERIMENTAL RESUTLS}

In this section we evaluate the methodology proposed in section III employing our indoor laboratory experimental testbed : multirobot Coherent Structure Testbed (mCoSTe). The mCoSTe is an indoor laboratory experimental testbed that consists of three flow tanks and a fleet of two types of microautonomous surface vehicles: the mASV and the mASVf. The proposed tracking strategy is validated using a fleet of 


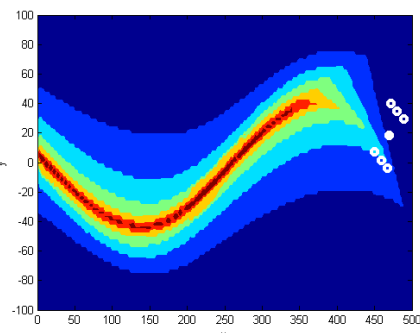

(a) $t=50 \mathrm{~s}$

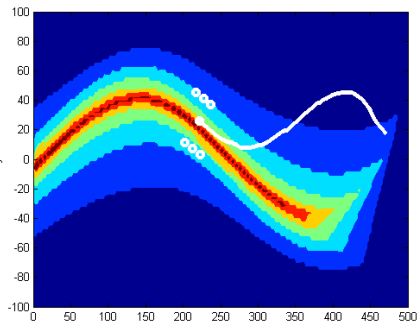

(e) $\mathrm{t}=98 \mathrm{~s}$

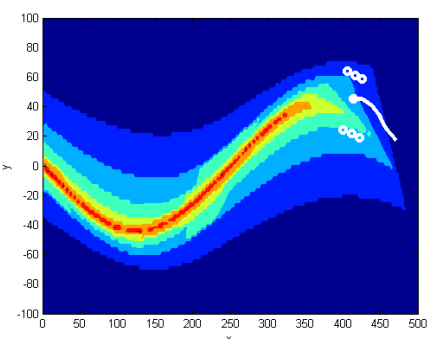

(b) $t=62 \mathrm{~s}$

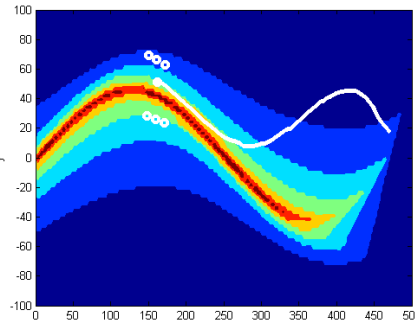

(f) $\mathrm{t}=110 \mathrm{~s}$

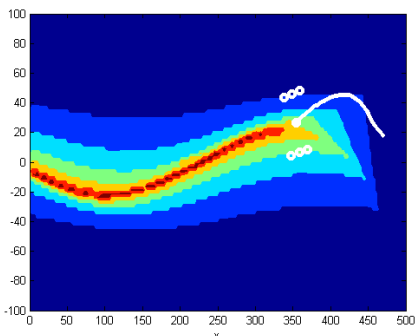

(c) $t=74 \mathrm{~s}$

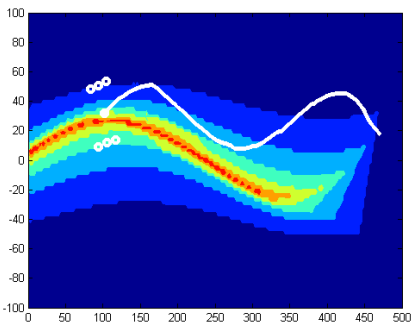

(g) $\mathrm{t}=122 \mathrm{~s}$

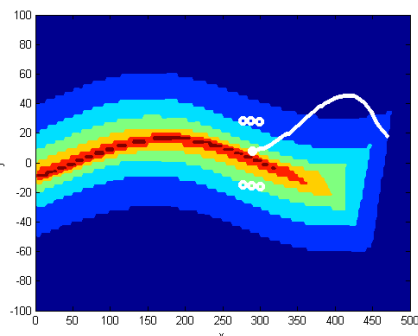

(d) $t=86 \mathrm{~s}$

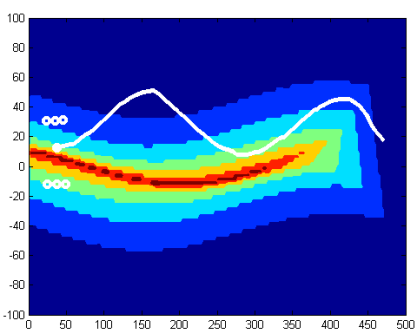

(h) $\mathrm{t}=134 \mathrm{~s}$

Fig. 5. The positions of the team of ASVs while tracking a time varying boundary in a conservative flow. The background shows the actual FTLE field calculated using complete knowledge of the flow field. The trajectory of $P_{c}$ is shown in white.

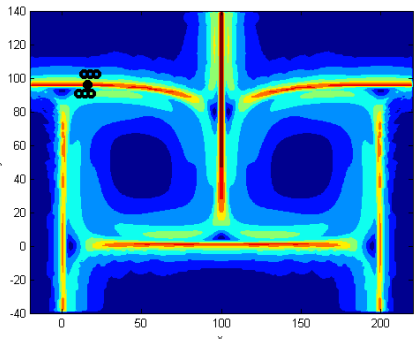

(a) $t=51 \mathrm{~s}$

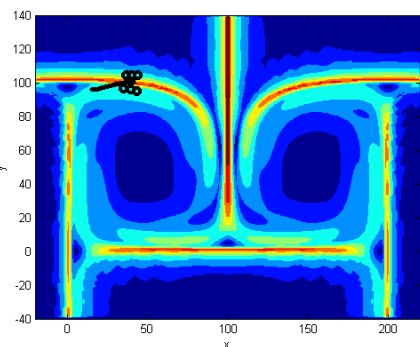

(b) $\mathrm{t}=62 \mathrm{~s}$

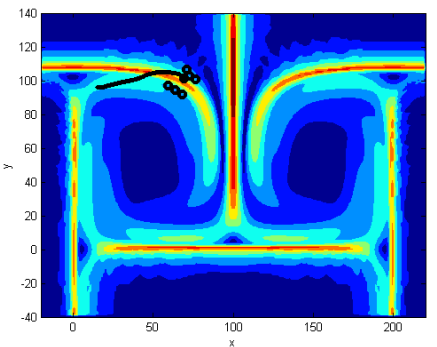

(c) $t=76 \mathrm{~s}$

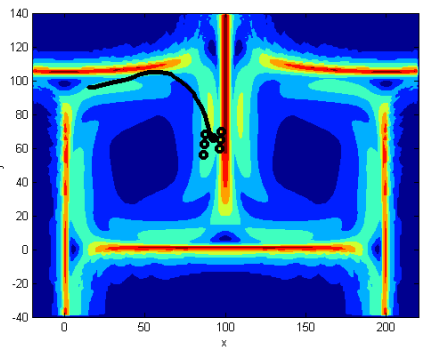

(d) $\mathrm{t}=88 \mathrm{~s}$

Fig. 6. The positions of the team of ASVs while tracking a time varying boundary in a wind-driven double-gyre flow model. The background shows the actual FTLE field calculated using complete knowledge of the flow field. The trajectory of $P_{c}$ is shown in black.

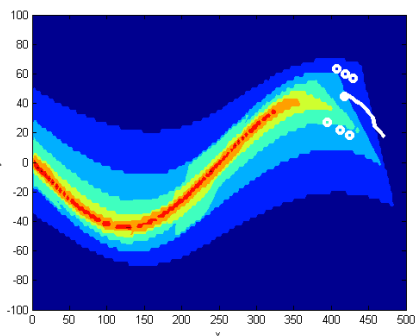

(a) $\mathrm{t}=62 \mathrm{~s}$

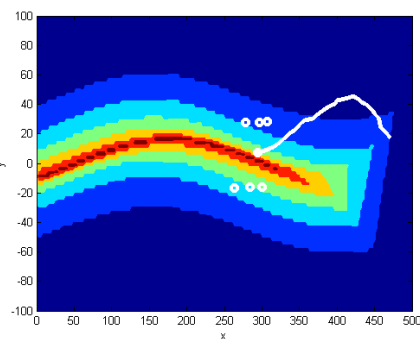

(b) $\mathrm{t}=86 \mathrm{~s}$

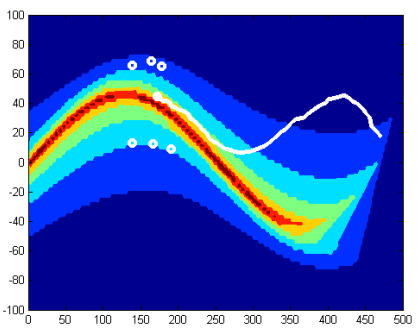

(c) $\mathrm{t}=110 \mathrm{~s}$

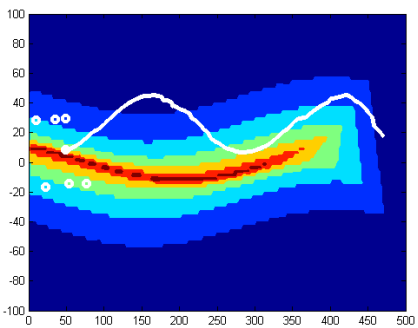

(d) $t=134 \mathrm{~s}$

Fig. 7. The positions of the team of ASVs while tracking a time varying boundary in a conservative flow n the presence of measurement noise.

mASVs in one of mCoSTe's flow tanks - the Multi Robot (MR) tank. The mASVs are differential drive surface vehicles equipped with a micro-controller board, XBee radio module, and an inertial measurement unit (IMU). The vehicles are approximately $12 \mathrm{~cm}$ long and have a mass of about $45 \mathrm{~g}$ each. Localization for the mASVs is provided by an external motion capture system. The MR tank which is $3 \times 3 \times 1 \mathrm{~m}^{3}$ in size, is designed to accommodate the operation of several mASVs and is able to create time-independent flow fields that exhibit kinematic and transport features similar to those observed in the ocean [11]. Flow fields are generated in the MR tank using rotating cylinders each having a diameter of $10 \mathrm{~cm}$. The speed and direction of the cylinders can be controlled individually to obtain a desired flow field in the MR tank. For additional 


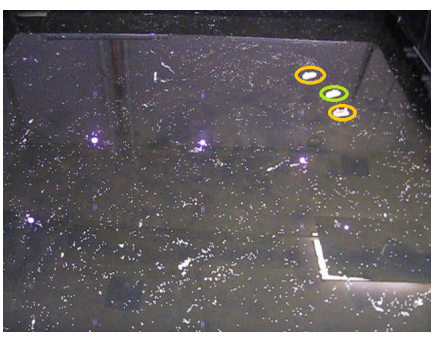

(a)

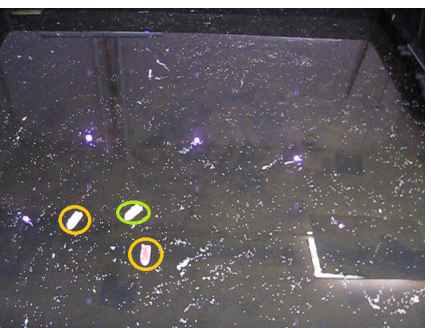

(c)

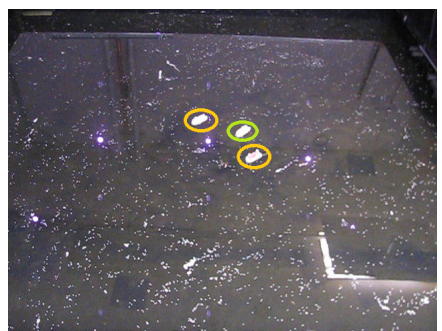

(b)

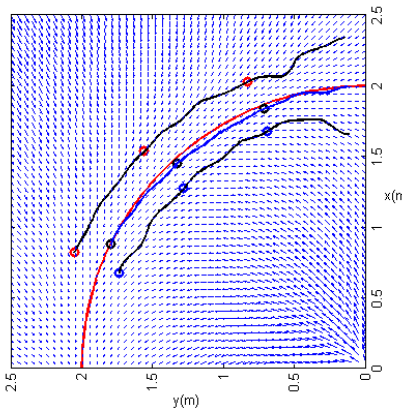

(d)
Fig. 8. (a)-(c) shows the motion of the mASVs in the MR tank. (d) shows the trajectories of the mASVs in the tank. The red line indicates the actual boundary, the blue line indicates the trajectory of $P_{c}$.

details about the various components of the mCoSTe and the quality of the flows that can be created using the mCoSTe, we refer the interested reader to [11].

In the first experiment, three mASVs along with four virtual ASVs were used to track a simulated static flow field of the form given in 23. The objective of this experiment was to demonstrate the viability of the proposed methodology on actual ASVs. The three mASVs were initially arranged in a saddle straddling formation with the center mASV $\left(P_{c}\right)$ tasked with tracking the boundary. Let the mASVs to the left and right of $P_{c}$ be denoted by $P_{l}$ and $P_{r}$ respectively. The virtual agents were placed at the four corners of the grid. For the flow field used for the experiment, $\lambda=0.133$ and $\beta$ was set to 0.005 , which from 22 indicates that the mASVs should converge to a distance of $0.194 \mathrm{~m}$ from the boundary. The actual average distance from the boundary to $P_{l}$ and $P_{r}$ were measured to be $0.183 \mathrm{~m}$ and $0.205 \mathrm{~m}$ respectively. Fig. 8(a)(c) show the motion of the mASVs in the MR tank and Fig. 8(d) show the trajectories of the mASVs.

In the next experiment, six flow cylinders were used to create an attracting coherent structure in the MR tank. The objective of this experiment was to evaluate the formation keeping strategy outlined in section III-B on an actual attracting manifold using three mASVs. In this case, the center mASV is provided the path of the attracting manifold beforehand. Fig. 9.(a)-(c) show the motion of the three mASVs along the attracting manifold and Fig. 9.d) shows the trajectories of the three mASVs in the tank. It can be seen that the mASVs maintain formation while moving along the boundary. It is not

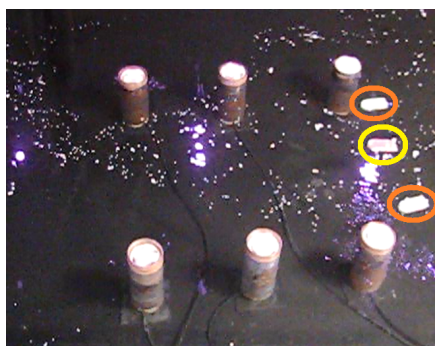

(a)

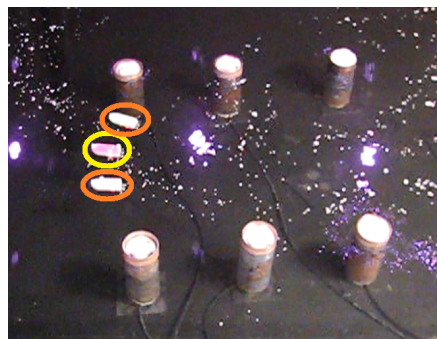

(c)

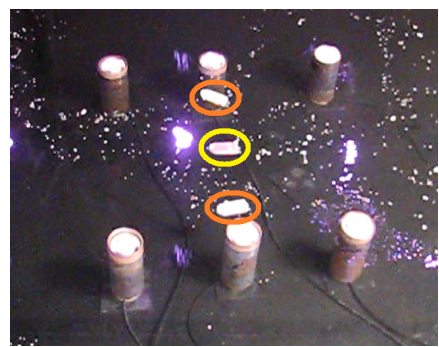

(b)

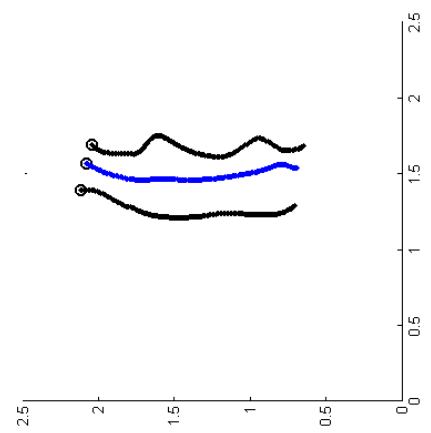

(d)
Fig. 9. (a)-(c) show the motion of the three mASVs along the attracting manifold. (d) shows the trajectories of the mASVs in the tank.

possible to provide a quantitative measure for the performance of the formation keeping strategy, since a method is currently unavailable to measure the flows in the MR tank accurately.

\section{Discussion AND FUTURE WORK}

In this work, we developed a methodology to track attracting LCS boundaries or unstable manifolds through the explicit onboard calculation of local FLTE fields. The proposed methodology was evaluated through a wide range of simulations and through experiments carried out in an indoor laboratory testbed. As shown in section V, the proposed boundary tracking and formation keeping strategies are robust when operating in static and time-varying conservative flows and even in the periodic wind-driven double-gyre flows. Even though the development and the analysis of the method does not explicitly consider measurement noise, the proposed method has been shown to work well with noisy measurements. This is not surprising since existing work [5, 4] suggest that a manifold tracking strategy based on FTLE computations should be robust to measurement noise. While the formation keeping strategy has been shown to work in the presence of noise, the effect of noise on its performance needs to be further analyzed and thus is a direction for future work. In addition, while the computations required for manifold tracking is currently carried out in a centralized manner, these computations can be easily distributed since the proposed strategy is based solely on local measurements of the flow field. Such a distributed implementation and validating its performance are areas for future work. 


\section{REFERENCES}

[1] Eric Forgoston, Lora Billings, Phillip Yecko, and Ira B. Schwartz. Set-based corral control in stochastic dynamical systems: Making almost invariant sets more invariant. Chaos, 21(013116), 2011.

[2] G. Haller. A variational theory of hyperbolic lagrangian coherent structures. Physica D, 240:574-598, 2011.

[3] G. Haller and G. Yuan. Lagrangian coherent structures and mixing in two-dimensional turbulence. Phys. D, 147:352-370, December 2000. ISSN 0167-2789. doi: 10.1016/S0167-2789(00)00142-1. URL http://dl.acm. org/citation.cfm?id=366463.366505.

[4] George Haller. Lagrangian coherent structures from approximate velocity data. Physics of Fluids, 14(6): 1851-1861, 2002.

[5] Cheryl S. Harrison and Gary A. Glatzmaier. Lagrangian coherent structures in the california current system sensitivities and limitations. Geophysical \& Astrophysical Fluid Dynamics, 106(1):22-44, 2012.

[6] Christoffer R. Heckman, M. Ani Hsieh, and Ira B. Schwartz. Controlling basin breakout for robots operating in uncertain flow environments. In International Symposium on Experimental Robotics (ISER 2014), Marrakech/Essaouira, Morocco, June 2014.

[7] M. A. Hsieh, S. Loizou, and V. Kumar. Stabilization of multiple robots on stable orbits via local sensing. In Proceedings of the International Conference on Robotics and Automation (ICRA) 2007, Rome, Italy, April 2007.

[8] M. A. Hsieh, E. Forgoston, T. W. Mather, and I. Schwartz. Robotic manifold tracking of coherent structures in flows. In Proc. IEEE Int. Conf. on Robotics and Automation (ICRA2012), Minneapolis, MN USA, May 2012.

[9] M. A. Hsieh, K. Mallory, and I. B. Schwartz. Distributed allocation of mobile sensing agents in geophysical flows. In Proc. of the 2014 American Controls Conference, Portland, OR, 2014.

[10] T. Inanc, S.C. Shadden, and J.E. Marsden. Optimal trajectory generation in ocean flows. In American Control Conference, 2005. Proceedings of the 2005, pages $674-$ 679, 8-10, 2005. doi: 10.1109/ACC.2005.1470035.

[11] D. Larkin, M. Michini, A. Abad, S. Teleski, and M. A. Hsieh. Design of the multi-robot coherent structure testbed (mcoste) for distributed tracking of geophysical fluid dynamics. In ASME International Design Engineering Technical Conferences (IDETC), Buffalo, NY USA, Aug 2014.

[12] T. Lolla, M. P. Ueckermann, P. Haley, and P. F. J. Lermusiaux. Path planning in time dependent flow fields using level set methods. In in the Proc. IEEE International Conference on Robotics and Automation, Minneapolis, MN USA, May 2012.

[13] K. Mallory, M. A. Hsieh, E. Forgoston, and I. B. Schwartz. Distributed allocation of mobile sensing swarms in gyre flows. Nonlin. Processes Geophys., 20
(5):657-668, 2013.

[14] M. Michini, M. A. Hsieh, E. Forgoston, and I. B. Schwartz. Robotic tracking of coherent structures in flows. IEEE Trans. on Robotics, 30(3):593-603, 2014.

[15] M. Michini, M. A. Hsieh, E. Forgoston, and I. B. Schwartz. Experimental validation of robotic manifold tracking in gyre-like flows. In Proc. IEEE/RSJ International Conference on Intelligent Robots and Systems (IROS) 2014, Chicago, IL USA, Sep 2014.

[16] M. Michini, H. Rastgoftar, M. A. Hsieh, and S. Jayasuriya. Distributed formation control for collaborative tracking of manifolds in flows. In Proc. of the 2014 American Control Conference (ACC 2014), Portland, OR, Jun 2014.

[17] H. E. Nusse and J. A. Yorke. A procedure for finding numerical trajectories on chaotic saddles. Physica $D$ Nonlinear Phenomena, 36:137-156, jun 1989. doi: 10.1016/0167-2789(89)90253-4.

[18] SCRIPPS. Naitonal HF RADAR network - surface currents, 2014. URL http://cordc.ucsd.edu/projects/ mapping/maps/

[19] C. Senatore and S.D. Ross. Fuel-efficient navigation in complex flows. In American Control Conference, 2008, pages $1244-1248$, june 2008. doi: 10.1109/ACC.2008. 4586663.

[20] Shawn C. Shadden, Francois Lekien, and Jerrold E. Marsden. Definition and properties of lagrangian coherent structures from finite-time lyapunov exponents in two-dimensional aperiodicflows. Physica D: Nonlinear Phenomena, 212(3-4):271 - 304, 2005. ISSN 0167-2789. doi: DOI:10.1016/j.physd.2005.10.007.

[21] A. Shchepetkin and J. McWilliams. Quasi-monotone advection schemes based on explicit locally adaptive dissipation. Monthly Weather Review, 126:1541-1580, 1998.

[22] A. F. Shchepetkin and J. C. McWilliams. The regional oceanic modeling system (roms): a split-explicit, freesurface, topography-following-coordinate oceanic model. Ocean Modeling, 9:347-404, 2005.

[23] R. Smith, A. M. Pereira, Y. Chao, P.P. Li, D. A. Caron, B. H. Jones, and G. Sukhatme. Autonomous underwater vehicle trajectory design coupled with predictive ocean models: A case study. In Proc. of the 2010 IEEE International Conference on Robotics and Automation, pages 4770-4777, Anchorage, AK, 2010.

[24] R. Smith, J. Kelly, and G. Sukhatme. Towards improving mission execution for autonomous gliders with an ocean model and kalman filter. In Proc. of the 2012 IEEE International Conference on Robotics and Automation, Minneapolis, MN, 2012.

[25] Ryan N. Smith, Yi Chao, Peggy P. Li, David A. Caron, Burton H. Jones, and Gaurav S. Sukhatme. Planning and implementing trajectories for autonomous underwater vehicles to track evolving ocean processes based on predictions from a regional ocean model. International Journal of Robotics Research, 29(12):1475-1497, 2010. 
doi: http://dx.doi.org/10.1177/0278364910377243.

[26] Ding Wang, Pierre F.J. Lermusiaux, Patrick J. Haley, Donald Eickstedt, Wayne G. Leslie, and Henrik Schmidt. Acoustically focused adaptive sampling and on-board routing for marine rapid environmental assessment. Journal of Marine Systems, 78:393-407, 2009. 